\title{
Structurisation and visualisation of design space for launch vehicle with hybrid rocket engine
}

\author{
Kazuhisa Chiba* \\ Graduate School of Informatics and Engineering, \\ The University of Electro-Communications, \\ 1-5-1, Chofugaoka, Chofu, Tokyo 182-8585, Japan \\ Email:kazchiba@uec.ac.jp \\ *Corresponding author
}

\section{Masahiro Kanazaki}

Graduate School of System Design,

Tokyo Metropolitan University,

6-6, Asahigaoka, Hino, Tokyo 191-0065, Japan

Email:kana@tmu.ac.jp

\section{Shin'ya Watanabe}

College of Information and Systems,

Muroran Institute of Technology,

27-1, Mizumotocho, Muroran, Hokkaido 050-8585, Japan

Email: sin@csse.muroran-it.ac.jp

\section{Koki Kitagawa and Toru Shimada}

Institute of Space and Astronautical Science,

Japan Aerospace Exploration Agency,

3-1-1, Yoshinodai, Chuo, Sagamihara 252-5210, Japan

Email: kitagawa.koki@jaxa.jp

Email: shimada.toru@jaxa.jp

\begin{abstract}
A single-stage launch vehicle with hybrid rocket engine has been conceptually designed by using design informatics, which has three points of view, i.e., problem definition, optimisation, and data mining. The primary objective of the present design is that the down range and the duration time in the lower thermosphere are sufficiently secured for the aurora scientific observation, whereas the initial gross weight is held down to the extent possible. The multidisciplinary design optimisation was performed by using a hybrid evolutionary computation. Data mining was also implemented by using a self-organising map and the stratum-type association analysis. Polypropylene and liquid oxygen with swirling flow are adopted as solid fuel and liquid oxidiser, respectively. The condition of single-time ignition is assumed in flight sequence. Consequently, the design information regarding the tradeoffs, the behaviours of the design variables in the design space to become the non-dominated solutions, and the implication of the design variables for the objective functions have been obtained in order to quantitatively differentiate
\end{abstract}


the advantage of hybrid rocket engine. The structurisation of the design space has been implemented in order to observe the effectiveness of the local regions of each design variable.

Keywords: design informatics; evolutionary computation; data mining; self-organising map; SOM; stratum-type association analysis; single-stage launch vehicle; hybrid rocket engine.

Reference to this paper should be made as follows: Chiba, K., Kanazaki, M., Watanabe, S., Kitagawa, K. and Shimada, T. (2016) 'Structurisation and visualisation of design space for launch vehicle with hybrid rocket engine', Int. J. Automation and Logistics, Vol. 2, Nos. 1/2, pp.26-44.

Biographical notes: Kazuhisa Chiba received his $\mathrm{PhD}$ in Engineering at Tohoku University, Japan. He is an Associate Professor at the University of Electro-Communications. His research interests are creation of novel aerospace vehicles using design informatics including issues related to evolutionary computations, data mining, and aerospace engineering.

Masahiro Kanazaki received his PhD in Information at the Tohoku University, Japan. He is an Associate Professor at the Tokyo Metropolitan University. His research interests include issues related to computational analysis of aerodynamics, flight dynamics and so on, to optimum design using evolutionary computations, and to their applications to aerospace engineering.

Shin'ya Watanabe received his $\mathrm{PhD}$ in Engineering at the Doshisha University, Japan. He is an Associate Professor at the Muroran Institute of Technology. His research interests are related to evolutionary computations, optimum design, and data mining.

Koki Kitagawa received his $\mathrm{PhD}$ in Engineering at the Tokyo Metropolitan Institute of Technology, Japan. He is an Assistant Professor at the Japan Aerospace Exploration Agency. His research interests are related to propulsion and combustion on solid and hybrid rockets.

Toru Shimada received his $\mathrm{PhD}$ in Engineering at the University of Tokyo, Japan. He is a Professor at the Japan Aerospace Exploration Agency. His research interests include issues related to gas dynamics, computational fluid dynamics, and space propulsion dynamics. He obtains several responsibilities of international committees regarding rocket and propulsion.

This paper is a revised and expanded version of a paper entitled 'Structurization of design space for launch vehicle with hybrid rocket engine using stratum-type association analysis' presented at The 18th Asia-Pacific Symposium on Intelligent and Evolutionary Systems (IES2014), Singapore, 10-12 November 2014.

\section{Introduction}

Design informatics is essential for practical design problems. Although solving design optimisation problems is important under the consideration of many disciplines of engineering (Arias-Montano et al., 2012), the most significant part of the process is the 
extraction of useful knowledge of the design space from results of optimisation runs. The results produced by multiobjective optimisation (MOO) are not an individual optimal solution but rather an entire set of optimal solutions due to tradeoffs. That is, the result of an MOO is not sufficient from the practical point of view as designers need a conclusive shape and not the entire selection of possible optimal shapes. On the other hand, this set of optimal solutions produced by an evolutionary MOO algorithm can be considered a hypothetical design database for design space. Then, data mining techniques can be applied to this hypothetical database in order to acquire not only useful design knowledge but also the structurisation and visualisation of design space for the conception support of basic design. This approach was suggested as design informatics (Chiba et al., 2012). The goal of this approach is the conception support for designers in order to materialise innovation. This methodology is constructed by the three essences as

1 problem definition

2 efficient optimisation

3 structurisation and visualisation of design space by data mining.

A design problem including objective function, design variable, and constraint, is strictly defined in view of the background physics for several months (problem definition is the most important process for all designers because it directly gives effect on the quality of design space. Since the garrulous objective-function/design-variable space including physics and design information which is not inherently necessary to consider should be performed unnecessary evolutionary exploration and mining, it is conceived to be low-quality design space), then optimisation is implemented in order to acquire non-dominated solutions (quasi-Pareto solutions) as hypothetical database. Data mining is performed for this database in order to obtain design information. Mining has the role as a post-process for optimisation. Mining result is the significant observations for next design phase and also becomes the material to redefine a design problem. Thereupon, a practical efficient technique as a self-organising map (SOM) is utilised in order to intuitively observe the correlations among the objective functions and the design variables. Moreover, the stratum-type association analysis has been applied in order to structurise and visualise the design space.

Intelligent and evolutionary systems including design informatics mentioned above have the significance for not only the systems themselves but also their applications to practical problems in order that science contributes toward the real world. Results themselves do not possess versatility in application problems due to their particularity. The versatility of a system is indeed critical in application problems because it is revealed that application range is expanded. Furthermore, the application results indicate the guidance for the improvement of systems.

In the present study, a single-stage launch vehicle with hybrid rocket engine using solid fuel and liquid oxidiser for the scientific observation of aurora will be conceptually designed by using design informatics approach. The final objective is that the advantage of re-ignition in the science mission for aurora observation on hybrid rocket will be quantitatively revealed. As a first step, an optimisation problem on single-time ignition, which is the identical condition of the current solid rocket, was defined so as to obtain the design information (Chiba et al., 2013). As a second step, the implication of solid fuels in the performance of hybrid rocket was revealed because the regression rate is one of the key elements for the performance of hybrid rocket (Chiba et al., 2014). Finally, the 
sequence using multi-time ignition, which is the advantage of hybrid rocket, will be investigated in order to reveal the ascendancy of hybrid rocket for aurora observation. This study corresponds to the additional extraction of the design information for the above first step. This study is a milestone to observe the quantitative difference of performance regarding ignition time.

\section{Design informatics}

\subsection{Optimisation method}

Design informatics after the definition of detailed problem is constructed by two phases as optimisation and data mining. Evolutionary computation is used for optimisation. Although a surrogate model (Keane, 2006) like as the Kriging model (Jeong et al., 2005), which is a response surface model developed in the field of spatial statistics and geostatistics, can be employed as optimisation method, it will not be selected because it is difficult to deal with a large number of design variables. In addition, since the designers require to present many exact optimum solutions for the decision of a compromise one, an evolutionary-based Pareto approach as an efficient multi-thread algorithm, which the plural individuals are parallel conducted, is employed instead of gradient-based methods. The optimiser used in the present study is the hybrid evolutionary method between the differential evolution (DE) and the genetic algorithm (GA) (Chiba, 2013). Moreover, global design information is primarily essential in order to determine a compromise solution. The view of hybridisation is inspired by the evolutionary developmental biology (Arthur, 2002). When there is the evolution which the Darwinism cannot explain in the identical species, each individual might have a different evolutionary methodology. When the practical evolution is imitated for the evolutionary computation, the different evolutionary algorithms might ultimately be applied to each individual in population. The making performance of next generation for each methodology depends on not only their algorithms but also the quality of candidate of parent in the archive of non-dominated solutions. The present hybridisation is intended to improve the quality of candidate of parent by sharing the non-dominated solutions in the archive among each methodology. In the present study, the evolutionary hybrid optimisation methodology between DE and GA is employed. It was confirmed that this methodology had the high performance regarding the convergence and diversity, as well as the strength for noise (Chiba, 2013). Note that noise imitates the error on computational analyses and experiments and is described as the perturbation on objective functions. It is an important factor when the optimisation for practical engineering problem is considered.

First, multiple individuals are generated randomly as an initial population. Then, objective functions are evaluated for each individual. The population size is equally divided into sub-populations between DE and GA (although sub-population size can be changed at every generations on the optimiser, the determined initial sub-populations are fixed at all generations in the present study). New individuals generated by each operation are combined in next generation. The non-dominated solutions in the combined population are archived in common. It is notable that only the archive data is in common between DE and GA. The respective optimisation methods are independently performed in the present hybrid methodology. 
The present optimisation methodology is a real-coded optimiser (Oyama et al., 2001). Although GA is based on the real-coded non-dominated sorting GA (the elitist NSGA-II) (Deb et al., 2002), it is made several improvements in order to be progressed with the diversity of solutions. Fonseca's Pareto ranking (Fonseca and Fleming, 1993) and the crowding distance (Deb et al., 2002) are used for the fitness value of each individual. The stochastic universal sampling (Baker, 1985) is employed for parents selection. The crossover rate is $100 \%$. The principal component analysis blended crossover (PCABLX) (Takahashi and Kita, 2001) and the confidence interval-based crossover (CIX) using $L_{2}$ norm (Hervas-Martinez et al., 2002) are used because of the high performance for the convergence and the diversity as well as the strength for noise (Chiba, 2013). The subpopulation size served by GA is equally divided for these two crossovers. The mutation rate is set to be constant as the reciprocal of the number of design variables. For alternation of generations, the Best- $N$ selection (Deb et al., 2002) is used. DE is used as the revised scheme (Robic and Filipic, 2005) for MOO from $\mathrm{DE} / \mathrm{rand} / 1 /$ bin scheme. The scaling factor $F$ is set to be 0.5 . The present optimiser has the function of range adaptation (Sasaki and Obayashi, 2005), which changes the search region according to the statistics of better solutions, for all design variables. In the present study, the range adaptation is implemented at every 20 th generations.

\subsection{Data-mining technique}

\subsubsection{Self-organising map}

In the present study, a SOM (Kohonen, 1995) is selected as a data-mining technique because the primary objective of data mining is the acquisition of global design information in order to implement the structuring of design space. The previous study (Chiba and Obayashi, 2008) indicated that SOM extracted the global design information for whole design space. The distinguishing feature of SOM is the generation of a qualitative description. The advantage of this method includes the intuitive visualisation of two-dimensional coloured maps of design space using bird's-eye-views. As a result, SOM reveals the tradeoffs among objective functions. Moreover, SOM addresses the effective design variables and also reveals how a specific design variable gives effects on objective functions and other design characteristics. One SOM is coloured for one variable of objective function, design variable, and other characteristic value so that the coloration pattern is compared each other. Therefore, the data mining using SOM might have a disadvantage to overlook important correlation in the problem with a large number of objective functions and design variables. Since the present study has a total number of 10 at most among the design requirements, design variables, and other variables that the influence will be observed, SOM is sufficient for the data mining manner.

In the present study, SOMs are generated by using commercial software Viscovery SOMine 4.0 plus produced by Eudaptics, GmbH (Deboeck and Kohonen, 1998). The uniqueness of the map generated by SOMine is assured due to Kohonen's Batch SOM algorithm and search of the best-matching unit for all input data and the adjustment of weight vector near the best-matching unit. The decoding manner of SOM is briefly explained by using Figure 1. Figure 1 is assumed to be SOMs coloured by three objective functions on the minimisation problem of three objective functions. The generated SOM is made from hexagonal grid, which has the values of objective functions and design variables as vector quantity. Grids are distributed on two-dimensional rectangular surface 
by the affinity of each objective-function value. Thereupon, the grids with high affinity of each objective-function value clusters around a grid. There is no physical import on the vertical and horizontal lines of SOM. The comparison among SOMs to be coloured by each vector quantity in each grid intuitively reveals the correlations among each vector quantity. There is similar coloration pattern between SOMs for $f_{1}$ and $f_{2}$ shown in Figure 1. This comparison shows that one objective function absolutely has low-value, when another objective function has low-value. Moreover, one objective function absolutely has high value, when another objective function has high value. That is, this comparison indicates that there is no tradeoff between $f_{1}$ and $f_{2}$. On the other hand, $f_{3}$ absolutely becomes large, when $f_{1}$ becomes small, and vice versa. This comparison indicates that there is severe tradeoff between $f_{1}$ and $f_{3}$.

Figure 1 Comparison example of coloured SOMs for minimisation problem with three objective functions as $f_{1}, f_{2}$, and $f_{3}$ (see online version for colours)

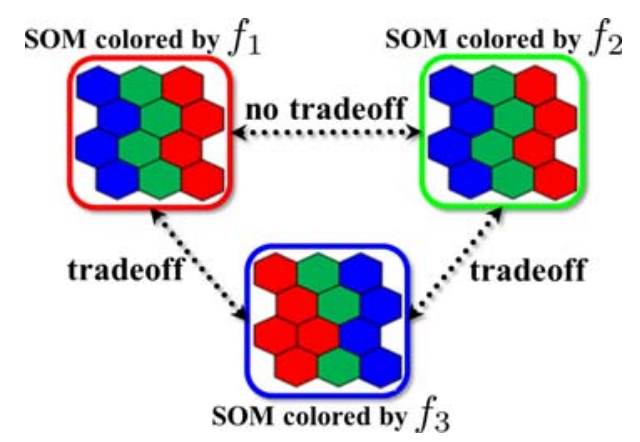

Note: Red describes high value and blue is low one.

\subsubsection{Stratum-type association analysis}

The data mining technique named as the stratum-type association analysis (Watanabe et al., 2014) is applied to analysing non-dominated solutions. The previous study (Chiba and Obayashi, 2008) employed the rough set theory in order to obtain the concrete rule regarding the design principles of design variables. Since the rough set theory gives individual rules based on the machine learning, it does not reveal the correlation knowledge among them. The present methodology systematises individual rules and structurise design space using a hierarchical dendrogram so that methodology obtains a bird's-eye view of it in order to have useful knowledge. The feature of the present methodology is a recursive clustering using association rules and a multi-granular analysis. The results of the recursive clustering can be visualised as a hierarchical dendrogram in which each node is a sub cluster of non-dominated solutions. The present system is expected to extract design information from a microscopic to a macroscopic view points due to the structurisation of design space.

- Procedure of system: First, the present system discretises continuous data for logical analysis. Second, association rules are derived from discretised data through logical analysis. Since designer would like to primarily acquire the design knowledge regarding objective function because it corresponds to design requirement. The association rules regarding objective function will be extracted at antecedent and 
consequent processes. The association rules are gradually integrated into subsets regarding the degree of coincidence at antecedent and consequent processes. Finally, the present methodology constructs a structured hierarchical dendrogram by clustering subsets based on synthetic correlations. It is essential that the methodology generates the subsets of non-dominated solutions using not the similarity among them but the inherent characteristics in each non-dominated solution.

- Clustering method for association rules: A hierarchical dendrogram can be simply generated through clustering the association rules with similarity and a multi-granular analysis. Therefore, the present methodology can be optionally selected regarding clustering manner. The methodology integrates the generated rules into subsets using not similarity but the accordance with the coincidence of the rules generated by the antecedent and consequent operations. A generated hierarchical dendrogram is simply constructed by the inclusive correlation among the subsets. Indeed, all elements which contain all extracted association rules are selected. All combinations of these elements are generated without overlap. Then, the subsets are generated in order to concentrate the elements. Thereupon, all of the subsets have common characteristics. That is, all nodes of a hierarchical dendrogram have one common characteristic at least. The characteristic in a node is useful knowledge for designers.

Table 1 Limitation of upper/lower values of each design variable

\begin{tabular}{lcc}
\hline Serial number & Design variable & Design space \\
\hline $\mathrm{dv} 1$ & Initial mass flow of oxidiser & $1.0 \leq \dot{m}_{\text {oxi }}(0)[\mathrm{kg} / \mathrm{sec}] \leq 30.0$ \\
$\mathrm{dv} 2$ & Fuel length & $1.0 \leq L_{\mathrm{fuel}}[\mathrm{m}] \leq 10.0$ \\
$\mathrm{dv} 3$ & Initial radius of port & $0.01 \leq r_{\mathrm{port}}(0)[\mathrm{m}] \leq 0.30$ \\
$\mathrm{dv} 4$ & Combustion time & $10.0 \leq t_{\mathrm{burn}}[\mathrm{sec}] \leq 40.0$ \\
$\mathrm{dv} 5$ & Initial pressure in combustion chamber & $3.0 \leq P_{c c}(0)[\mathrm{MPa}] \leq 6.0$ \\
$\mathrm{dv} 6$ & Aperture ratio of nozzle & $5.0 \leq \epsilon[-] \leq 8.0$ \\
$\mathrm{dv} 7$ & Elevation at launch time & $50.0 \leq \phi(0)[\mathrm{deg}] \leq 90.0$ \\
\hline
\end{tabular}

Figure 2 Conceptual illustrations of hybrid rocket and its design variables regarding the geometry (see online version for colours)
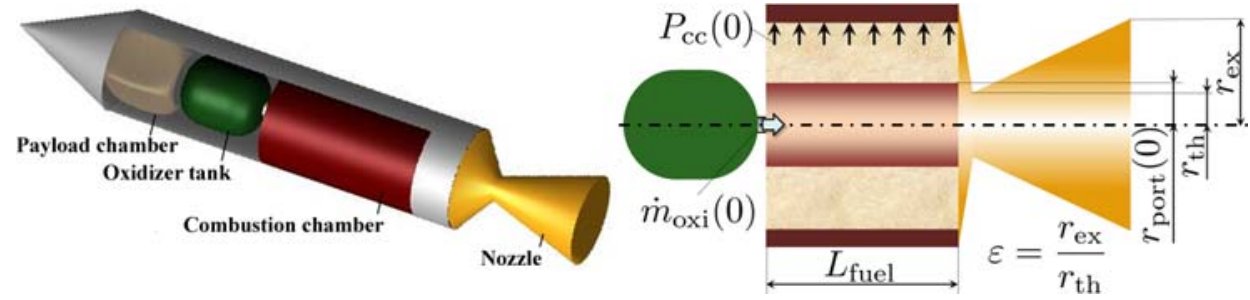

Note: Aperture ratio of nozzle $\epsilon$ is described by using the radius at nozzle exit $r_{\mathrm{ex}}$ and the radius at nozzle throat $r_{\mathrm{th}}$. 


\section{Problem definition}

Single-stage rockets have been researched and developed for the scientific observations and the experiments of high-altitude zero-gravity condition, whereas multi-stage rockets have been also studied for the orbit injection of payload. The Institute of Space and Astronautical Science (ISAS), Japan Aerospace Exploration Agency (JAXA) has been operating $\mathrm{K}$ (Kappa), L (Lambda), and $\mathrm{M}(\mathrm{Mu})$ series rockets as the representatives of solid rocket in order to contribute to the space scientific research. A lower-cost and more efficient rocket is necessary due to the retirement of $\mathrm{M}-\mathrm{V}$ in 2008 and in order to promote space scientific research. In fact, E (Epsilon) rocket began to be operated from September 2013. On the other hand, the launch vehicle with hybrid rocket engine using solid fuel and liquid oxidiser has been researched and developed as an innovative technology in mainly Europe and the USA (Karabeyoglu, 2013; Simurda et al., 2013). The present study will investigate the conceptual design in order to develop a next-generation single-stage launch vehicle with hybrid rocket engine. Since the technologies of hybrid rocket engine for single-stage and multi-stage are not independent, the solution of the fundamental physics regarding single-stage hybrid rocket is diverted to multi-stage one. A hybrid rocket offers the several advantages as higher safety, lower cost, and pollution free flight. The multi-time ignition is the especial ascendancy of hybrid rocket engine (Saraniero et al., 1973). On the other hand, the disadvantage of a hybrid rocket engine is in its combustion. As a hybrid rocket engine has low regression rate of solid fuel due to turbulent boundary layer combustion, the thrust of hybrid rocket engine is less than that of pure solid and pure liquid engines which can obtain premixed combustion (Karabeyoglu et al., 2002). In addition, as the mixture ratio between solid fuel and liquid oxidiser is temporally fluctuated, thrust changes with time. Multidisciplinary design requirements should be considered in order to surmount the disadvantage of hybrid rocket engine. Moreover, exhaustive design information will be obtained in order to additionally consider productive and market factors for practical problems (it is difficult that optimisation deals with them due to the difficulty of the definition).

The conceptual design for a single-stage hybrid rocket, simply composed of a payload chamber, an oxidiser tank, a combustion chamber, and a nozzle (Kosugi et al., 2011) shown in Figure 2, is considered in the present study. A single-stage hybrid rocket for aurora scientific observation will be focused because the rocket for more efficient scientific observation is desired for successfully obtaining new scientific knowledge on the aurora observation by ISAS in 2009. In addition, a single-stage hybrid rocket problem fits for the resolution of the fundamental physics regarding hybrid rocket engine and for the improvement of the present design problem due to its simplification.

\subsection{Objective functions}

Three objective functions are defined in the present study. First objective is the maximisation of the down range in the lower thermosphere (altitude of 90 to $150[\mathrm{~km}]$ ) $R_{d}$ [km] (obj1). Second is the maximisation of the duration time in the lower thermosphere $T_{d}$ [sec] (obj2). It recently turns out that atmosphere has furious and intricate motion in the lower thermosphere due to the energy injection, which leads aurora, from high altitude. The view of these objective functions is to secure the horizontal distance and time for the competent observation of atmospheric temperature 
and the wind for the elucidation of atmospheric dynamics and the balance of thermal energy. Third objective is the minimisation of the initial gross weight of launch vehicle $M_{\mathrm{tot}}(0)[\mathrm{kg}](\mathrm{obj} 3)$, which is generally the primary proposition for space transportation system.

\subsection{Design variables}

Seven design variables are used as initial mass flow of oxidiser $\dot{m}_{\mathrm{oxi}}(0)[\mathrm{kg} / \mathrm{sec}](\mathrm{dv} 1)$, fuel length $L_{\text {fuel }}[\mathrm{m}](\mathrm{dv} 2)$, initial radius of port $r_{\text {port }}(0)[\mathrm{m}](\mathrm{dv} 3)$, combustion time $t_{\text {burn }}[\mathrm{sec}](\mathrm{dv} 4)$, initial pressure in combustion chamber $P_{\mathrm{cc}}(0)[\mathrm{MPa}](\mathrm{dv} 5)$, aperture ratio of nozzle $\epsilon[-](\mathrm{dv} 6)$, and elevation at launch time $\phi[\mathrm{deg}](\mathrm{dv} 7)$. The design variables regarding the geometry of launch vehicle are visualised in Figure 2. Note that there is no constraint except the limitations of upper/lower values of each design variable summarised in Table 1. These upper/lower values are exhaustively covering the region of design space which is physically admitted. When there is a sweet spot (the region that all objective functions proceed optimum directions) in the objective-function space, the exploration space would intentionally become narrow due to the operation of range adaptation on the evolutionary computation.

\subsection{Evaluation method}

First of all, the mixture ratio between liquid oxidiser and solid fuel $O / F(t)$ is computed by the following equation.

$$
\begin{aligned}
O / F(t) & =\frac{\dot{m}_{\text {oxi }}(t)}{\dot{m}_{\text {fuel }}(t)} . \\
\dot{m}_{\text {fuel }}(t) & =2 \pi r_{\text {port }}(t) L_{\text {fuel }} \rho_{\text {fuel }} \overline{\dot{r}}_{\text {port }}(t), \\
r_{\text {port }}(t) & =r_{\text {port }}(0)+\int \dot{r}_{\text {port }}(t) d t .
\end{aligned}
$$

$\dot{m}_{\text {oxi }}(t)$ and $\dot{m}_{\text {fuel }}(t)$ are the mass flow of oxidiser [kg/sec] and the mass flow of fuel $[\mathrm{kg} / \mathrm{sec}]$ at time $t$, respectively. $r_{\text {port }}(t)$ is the radius of port [m] at $t, L_{\text {fuel }}$ describes fuel length, and $\rho_{\text {fuel }}$ is the density of fuel $\left[\mathrm{kg} / \mathrm{m}^{3}\right] \dot{r}_{\text {port }}(t)$ describes the regression rate. After that, an analysis of chemical equilibrium is performed by using NASA-chemical equilibrium with applications (CEA) (Gordon and McBride, 1994), then trajectory, thrust, aerodynamic, and structural analyses are respectively implemented. The present rocket is assumed as a point mass. As the time step is set to be 0.5 [sec] in the present study, it takes roughly $10[\mathrm{sec}]$ for the evaluation of an individual using a general desktop computer.

A combustion chamber is filled with solid fuel with a single port at the centre to supply oxidiser. As the regression rate to the radial direction of the fuel $\dot{r}_{\text {port }}(t)[\mathrm{m} / \mathrm{sec}]$ generally governs the thrust power of hybrid rocket engine, it is a significant parameter. The following experimental model (Hirata et al., 2011; Yuasa et al., 2012) is used in the present study. 


$$
\begin{aligned}
\dot{r}_{\text {port }}(t) & =a_{\text {fuel }} \times G_{\text {oxi }}^{n_{\text {fuel }}(t)} \\
& =a_{\text {fuel }} \times\left(\frac{\dot{m}_{\text {oxi }}(t)}{\pi r_{\text {port }}^{2}(t)}\right)^{n_{\text {fuel }}},
\end{aligned}
$$

where $G_{\text {oxi }}(t)$ is oxidiser mass flux $\left[\mathrm{kg} / \mathrm{m}^{2} / \mathrm{sec}\right] . a_{\text {fuel }}[\mathrm{m} / \mathrm{sec}]$ and $n_{\text {fuel }}[-]$ are the constant values experimentally determined by fuels. In the present study, liquid oxygen as liquid oxidiser and polypropylene as thermoplastic resin for solid fuel in order to adopt swirling flow for the supply mode of oxidiser. Therefore, $a_{\text {fuel }}$ and $n_{\text {fuel }}$ are respectively set to be $8.26 \times 10^{-5}[\mathrm{~m} / \mathrm{sec}]$ and 0.5500 .

\section{Results}

\subsection{Optimisation result}

The population size is set to be 18 and evolutionary computation is performed until 3,000 generations. The plots of acquired non-dominated solutions are shown in Figure 3, which reveals that the connecting and convex non-dominated surface except several isolated individuals is generated.

Figure 3 Plots of non-dominated solutions derived by optimisation, (a) plotted in three-dimensional objective-function space (red) and their plots projected onto two dimensions (b) plots projected onto two-dimension between down range $R_{d}(\mathrm{obj} 1)$ and duration time $T_{d}$ (obj2) (light green) (c) plots projected onto two-dimension between duration time $T_{d}$ (obj2) and initial gross weight $M_{\mathrm{tot}}(0)(\mathrm{obj} 3)$ (light blue) (d) plots projected onto two-dimension between down range $R_{d}$ (obj1) and initial gross weight $M_{\text {tot }}(0)($ obj3) (blue) (see online version for colours)

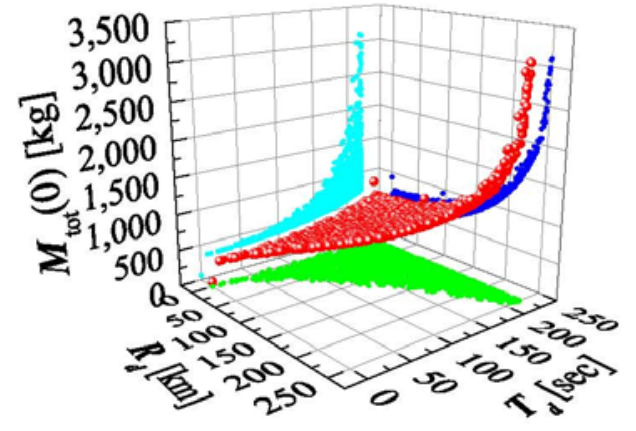

(a)

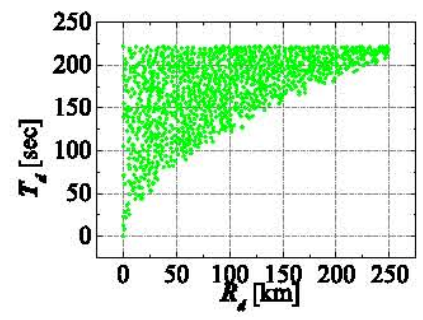

(b)

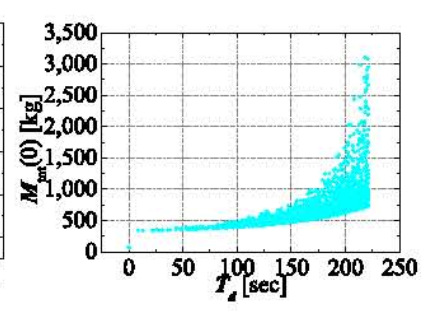

(c)

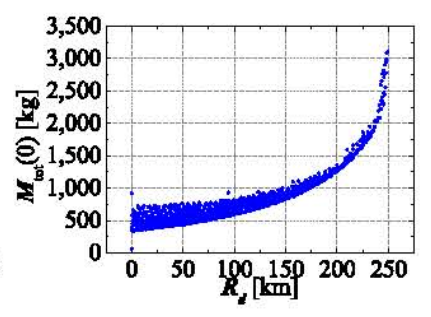

(d) 
There is no tradeoff between the down range $R_{d}$ and the duration time $T_{d}$ in the lower thermosphere shown in Figure 3(b). Figure 3 also shows that there are upper limits of roughly $250[\mathrm{~km}]$ for $R_{d}$ and of roughly 220 [sec] for $T_{d}$. Therefore, the projection plots onto two-dimension between $R_{d}$ and $T_{d}$ do not converge in one point. In the present study, the initial mass flow of oxidiser $\dot{m}_{\text {oxi }}(0)$ (dv1) has the limitation of upper/lower values.

Since the regression rate of fuel $\dot{r}_{\text {port }}(t)$ as an experimental model uses the mass flow of oxidiser $\dot{m}_{\text {oxi }}(t), \dot{r}_{\text {port }}(t)$ has constraints. As a result, the upper limits are generated for $R_{d}$ and $T_{d}$.

There is an incomplete tradeoff between $T_{d}$ and the initial gross weight $M_{\mathrm{tot}}(0)$ shown in Figure 3(c). The convex non-dominated surface to optimum direction with incompleteness is generated due to the upper limit of $T_{d}$. As the inclination $\Delta M_{\mathrm{tot}}(0)=\Delta T_{d}$ is small on the convex curve, $T_{d}$ can be substantially improved when trifling $M_{\mathrm{tot}}(0)$ would be sacrificed. In addition, Figure 3(c) shows that the minimum $M_{\mathrm{tot}}(0)$ to reach the upper limit of $T_{d}$ (roughly $220[\mathrm{sec}]$ ) is approximately $700[\mathrm{~kg}]$. And also, the smallest $M_{\text {tot }}(0)$ to attain to the lower thermosphere (altitude of $90[\mathrm{~km}]$ ) is approximately $350[\mathrm{~kg}]$. As these values are better than those of the solid rockets which are operated at present for scientific observation, it suggests that hybrid rocket has an advantage even when hybrid rocket does not have a sequence of multi-time ignition.

There is a severe tradeoff between $R_{d}$ and $M_{\mathrm{tot}}(0)$ shown in Figure 3(d) (although the down range strictly has the upper limit, it seems that the clean convex curve is generated because the upper limit is on the edge of the non-dominated surface). Figure 3 shows that the maximum $R_{d}$ is roughly $130[\mathrm{~km}]$ when the minimum $M_{\mathrm{tot}}(0)$ to reach the upper limit of $T_{d}$ (roughly $700[\mathrm{~kg}]$ ) is adopted. $M_{\mathrm{tot}}(0)$ should be absolutely increased in order to have more $R_{d}$ (greater than $130[\mathrm{~km}]$ ) despite no increase of $T_{d}$ (remaining roughly 220 [sec]). This fact suggests that the design strategies for the maximisations of $R_{d}$ and $T_{d}$ are different.

\subsection{Data-mining result}

\subsubsection{Knowledge obtained by using SOM}

Figure 4 shows SOMs coloured by the objective functions and the design variables. As this SOM learning is implicated based on the values of the objective functions as the indicator for the similarity on the neural network, SOMs coloured by the objective functions have absolutely gradation shown in Figure 4(a). SOMs coloured by the design variables are shown in Figure 4(b). The upper/lower values of colouring range are set to be the upper/lower values of each design variable defined in the problem summarised in Table 1. Figure 4(c) also shows SOM coloured by the design variables. The upper/lower values of colouring range in Figure 4(c) are set to be maximum/minimum values of each design variable in non-dominated solutions. Since the evolutionary exploration is performed for the design space, the range for the respective design variables in Figure 4(c) becomes absolutely narrow compared with Figure 4(b). 
Figure 4 SOMs generated by the objective-function values, (a) coloured by the objective functions (b) coloured by the design variables. The colour range is set to be their upper/lower values in the problem definition in Table 1 (c) coloured by the design variables. The colour range is set to be the $\mathrm{max} / \mathrm{min}$ values in non-nominated solutions (see online version for colours)

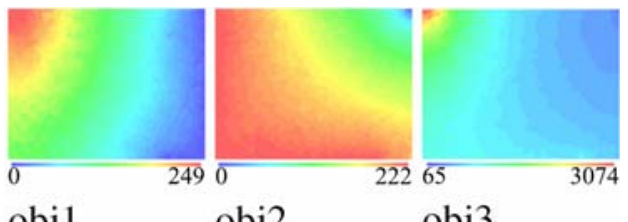

$$
\text { obj1 obj2 obj3 }
$$

(a)

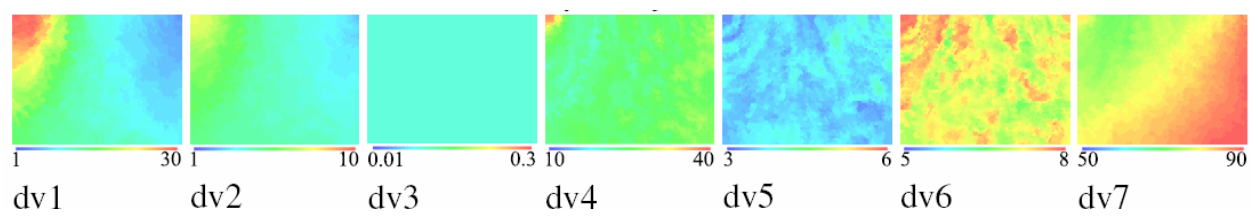

(b)
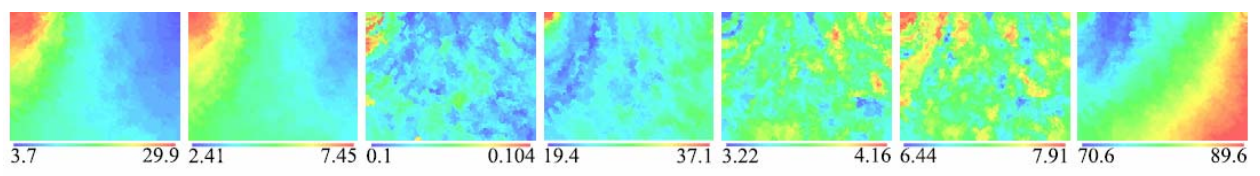

dv 1

dv2

dv3

dv4

dv5 dv6

dv7

(c)

- Acquired information regarding relationship among objective functions: The comparison of the colouring pattern in Figure 4(a) reveals the tradeoffs among the objective functions. When obj1 is high value (red region), obj2 absolutely becomes high. However, as obj1 does not always become high whenever obj2 is high, this relationship is irreversible. This is because not only $R_{d}$ (obj1) but also the attained maximum altitude gives the effect on $T_{d}$ (obj2). In contrast, when obj2 is low value (blue region), obj1 absolutely becomes low. However, as obj2 does not always become low whenever obj1 is low, this relationship is similarly irreversible.

Although there is no tradeoff in the global space of the objective functions, there is locally tradeoff at the right bottom of Figure 4(a). This local tradeoff is caused by the upper limit of $T_{d}$ (obj2), that is, reaching the maximum value of $T_{d}$ (obj2) is easier than achieving the maximum value of $R_{d}(\mathrm{obj} 1)$.

Since $M_{\mathrm{tot}}(0)$ (obj3) is the minimisation function, there are severe tradeoffs between obj3 and the others. It especially reveals the severe problem that the optimum direction of $R_{d}(\mathrm{obj} 1)$ and the pessimum direction of $M_{\mathrm{tot}}(0)$ (obj3) accord (observing the upper left on SOM regarding obj1 and obj2 reveals that the better obj1 and the worse obj3 accord). The structural constraints and the combustion mode should be reconsidered in order to avoid the condition which the optimum direction of obj1 and the pessimism direction of obj3 accord. On the other hand, the optimum region of $T_{d}$ (obj2) and the pessimum direction of $M_{\mathrm{tot}}(0)$ (obj3) overlap only in part.

Therefore, $M_{\text {tot }}(0)$ can become low when $T_{d}$ is the primary objective. On the other 
hand, the minimum $M_{\text {tot }}(0)$ is decided by the expected $R_{d}$ when $R_{d}$ is the primary objective, that is, the minimum initial gross weight depends on the mission requirement as the necessary down range.

- Acquired information regarding behaviour of design variables: Figure 4(b) reveals the behaviour of each design variable in the design space with the defined wide range which is physically available in order to become the non-dominated solutions.

All non-dominated solutions have higher dv1 than the lower bound of dv1 defined in Table 1 because the first SOM from the left column in Figure 4(b) does not have the colour of deep blue. This fact suggests that the minimum initial mass flow of oxidiser is necessary in order to attain to the lower thermosphere (altitude of $90[\mathrm{~km}])$. The mass flow of oxidiser $\dot{m}_{\text {oxi }}(t)$ affects the structural weight because of the increase of the filling pressure of oxidiser tank and the pressure of the combustion chamber $P_{\mathrm{cc}}(t)$. Thereupon, the initial mass flow of oxidiser $\dot{m}_{\text {oxi }}(0)$ (dv1) is essential in order to improve $M_{\mathrm{tot}}(0)$ (obj3).

The value of dv2 does not have both high and low because the second SOM from the left column in Figure 4(b) does not have the colour of red and deep blue. As the minimum fuel length is necessary in order to attain to the lower thermosphere (altitude of $90[\mathrm{~km}]$ ), dv2 does not have low. On the other hand, as it is considerable that the fuel length $L_{\text {fuel }}(\mathrm{dv} 2)$ does not affect strongly on the maximisation of $R_{d}$ and $T_{d}$ rather than the initial mass flow of oxidiser $\dot{m}_{\text {oxi }}(0)(\mathrm{dv} 1), L_{\text {fuel }}(\mathrm{dv} 2)$ does not have high value.

The third SOM from the left column in Figure 4(b) reveals that the value of dv3 is roughly constant. This fact indicates that there is the optimum initial radius of port $r_{\text {port }}(0)(\mathrm{dv} 3)$, which might be determined by the combustion mode and the regression rate. However, the initial radius of port does not depend on the fuels (Chiba et al., 2014). The influence of oxidiser-flow mode is an assignment because of the low-fidelity evaluation model in the present study.

The fourth SOM from the left column in Figure 4(b) reveals that the value of dv4 is in the narrow region of the design space. However, the combustion time $t_{\text {burn }}(\mathrm{dv} 4)$ becomes high when $M_{\text {tot }}(0)$ (obj3) is high (the upper left corners in the fourth SOM from the left column, where is the maximum direction of obj3, has high). That is, the combustion time $t_{\text {burn }}(\mathrm{dv} 4)$ has identical behaviour of the initial radius of port $r_{\text {port }}(0)$ (dv3) except the design space where it directly gives effect on $M_{\mathrm{tot}}(0)$ (obj3) (note that a variable directly affects when degrees of colouring transition between two SOMs are similar, for example, the upper left region of SOMs for obj3 in Figure 4(a) and for dv4 in Figure 4(b). On the other hand, it assumes that a variable indirectly affects when degrees of colouring transition between two SOMs are not similar, for example, the upper left region of SOMs for obj3 in Figure 4(a) and for dv1 in Figure $4(\mathrm{~b})$. The combustion time $t_{\text {burn }}(\mathrm{dv} 4)$ is a sensitive design variable for the characteristics of the regression rate (Chiba et al., 2014).

The third SOM from the right column in Figure 4(b) shows that the value of dv5 has low value. The high value of the initial pressure in combustion chamber $P_{\mathrm{cc}}(0)(\mathrm{dv} 5)$ fundamentally gives high thrust. As it is expected that the structural requirement is not fulfilled due to high pressure, the structural fulfilment should be confirmed by 
the parametric study regarding the structural safety factor. In addition, the time fluctuation regarding the pressure in combustion chamber $P_{\mathrm{cc}}(\mathrm{t})$ should be observed in the next-step design problem.

The second SOM from the right column in Figure 4(b) shows that the value of dv6 has the colouring pattern in a muddle which is similar to that of dv5, although there is the difference of colour. Since the aperture ratio of nozzle $\epsilon$ (dv6) becomes high in order to keep the high thrust, $\epsilon(\operatorname{dv} 6)$ indirectly give the effect on the objective functions. In fact, the high value of dv6 is on the upper left of SOM, which is the region to become high objective functions.

The first SOM from the right column in Figure 4(b) shows that the coloration pattern of SOM coloured by dv7 is similar to that by obj1. As the vertical launch would be implemented when the elevation at launch time $\phi(0)(\mathrm{dv} 7)$ becomes high, it is easily understandable that $R_{d}$ (obj1) is low.

- Influence of design variables on objective functions: Figure 4(c) reveals the influence direction of the design variables for the objective functions. And also, the correlations among the design variables are shown when the SOMs coloration pattern is compared between Figure 4(a) and Figure 4(c).

In the first place, the influence of each design variable on the maximisation of $R_{d}$ (obj1) will be summarised. The high-value region of dv1 is in concord with the high-value region of obj1. Since the high value of the initial mass flow of oxidiser $\dot{m}_{\text {oxi }}(0)(\mathrm{dv} 1)$ increases thrust, $R_{d}$ (obj1) is sensitive. The high-value region of $\mathrm{dv} 2$ is also in concord with the high-value region of obj1. The coloration pattern on SOM regarding dv2 is extremely similar to that regarding dv1. Fuel length $L_{\text {fuel }}(\mathrm{dv} 2)$ is one of the constitutional elements for the mass flow of the fuel $\dot{m}_{\text {fuel }}(t)$, that is, the mixture ratio between liquid oxidiser and solid fuel $O / F(t)$ strongly has the influence of $L_{\text {fuel }}(\mathrm{dv} 2)$. As the mixture ratio $O / F(t)$ is essential for the performance of hybrid rocket, the implication of $O / F(t)$ for $R_{d}$ (obj1) also shows its significance. As the coloration pattern on SOM regarding dv3 is not similar to that regarding obj1, $\mathrm{dv} 3$ does not give effects on the optimisation of obj1. The initial radius of port $r_{\text {port }}(0)(\mathrm{dv} 3)$ is not essential for the optimisation of $R_{d}$ (obj1) whenever the value of $r_{\text {port }}(0)(\mathrm{dv} 3)$ stays in the range to become non-dominated solutions. High value of dv4 achieves optimising obj1. However, the down range can be high even when combustion time $t_{\text {burn }}(\mathrm{dv} 4)$ is less than the threshold (roughly 27 [sec], it is revealed by the comparison between the first SOMs from the left in Figure 4(a) for each fuel and the fourth SOMs from the left in Figure 4(c). This is because the region on the upper left SOM is different coloration patterns between obj1 and dv4, and the transformation of colour on SOM regarding dv4 is sharp. Since the coloration pattern of SOM regarding dv5 is not in concord with that regarding obj1, there is no direct influence on obj1. As the pressure in combustion chamber $P_{\mathrm{cc}}(\mathrm{t})$ has the constraints regarding the structure mode and the safety factor, the initial pressure in combustion chamber $P_{\mathrm{cc}}(0)$ (dv5) would not have the flexibility to affect on $R_{d}$ (obj1). Therefore, the sensitivities of the structure mode and the safety factor should be investigated. As the coloration pattern of SOM regarding dv6 is similar to that regarding $d v 5$, it reveals that there is also no direct influence on obj1. Although the high value of $\operatorname{dv} 6$ tends to optimise obj $1, \operatorname{dv} 6$ is not essential. The aperture ratio of 
nozzle $\epsilon(\mathrm{dv} 6)$ is not uniquely determined for $R_{d}(\mathrm{obj} 1)$ and the other objective functions because the optimum aperture ratio of nozzle is fluctuated by the pressures in combustion chamber and flight altitude in order to be optimum expansion. Therefore, the aperture ratio of nozzle $\epsilon(\operatorname{dv} 6)$ does not directly have the influence on $R_{d}$. Although the value of $\mathrm{dv} 7$ should be low in order to optimise obj1, it should not be too low. Although $R_{d}$ (obj1) increases when the elevation $\phi(\operatorname{dv} 7)$ is low, the maximum attainable altitude decreases due to the projectile motion which is the assumed flight sequence. Therefore, multi-time ignition is essential in order that the down range is increased whereas the maximum attainable altitude keeps high.

In the second place, the influence on the maximisation of $T_{d}$ (obj2) will be summarised. The coloration pattern of all of the SOMs regarding the design variables is different from that of SOM regarding obj2. This difference depends on the manageable objective function due to its upper limit. The maximisation of obj2 is connotatively accompanied the maximisation of obj 1 . When the influence of the design variables on obj2 will be minutely investigated, the local non-dominated solutions on the convex surface in Figure 3(c) should be extracted in order that mining is separately performed for them.

In the third place, the influence on the minimisation of $M_{\mathrm{tot}}(0)(\mathrm{obj} 3)$ will be summarised. As the coloration pattern of SOMs right hand side regarding dv1 is in concord with that regarding obj3. This fact reveals that dv1-reduction is the necessary condition to minimise obj3. Since the volume of the installed oxidiser decreases when the initial mass flow of oxidiser $\dot{m}_{\text {oxi }}(0)(\mathrm{dv} 1)$ is reduced, the initial gross weight $M_{\text {tot }}(0)$ (obj3) is consequently reduced. As the initial mass flow of oxidiser $\dot{m}_{\text {oxi }}(0)$ (dv1) gives effect on the maximisation of $R_{d}$ (obj1), this design variable $\dot{m}_{\text {oxi }}(0)(\mathrm{dv} 1)$ covers the essential role in the present study. The coloration pattern of SOM regarding dv2 is similar to that regarding dv1. $M_{\mathrm{tot}}(0)(\mathrm{obj} 3)$ can be reasonably reduced when the fuel length $L_{\text {fuel }}(\mathrm{dv} 2)$ shortens. The coloured SOMs show that the values of $d v 3, d v 4, d v 5$, and dv6 are not directly effective on obj3. Although the high value of $\operatorname{dv} 7$ is a necessary condition to minimise obj3, the optimum direction is different between $\mathrm{dv} 7$ and obj3. Therefore, it is considerable that dv1 and dv2 directly give effects on obj3. When the elevation at launch time $\phi(0)$ (dv7) becomes high (in the vicinity of $90[\mathrm{deg}]$ ), the necessary volume of the fuel can be held down because the flight distance from the ground to the lower thermosphere (altitude of $90[\mathrm{~km}])$ shortens. $M_{\mathrm{tot}}(0)(\mathrm{obj} 3)$ consequently becomes low.

\subsubsection{Knowledge obtained by using stratum-type association analysis}

A discretisation is necessary for rule generation. The hybrid discretisation manner between an equivalent distance and an equivalent frequency method (Watanabe et al., 2014 ) is utilised. The number of discretisation is set to be 10 for the objective functions and the design variables in the present study. The value of minimum support depends on the generated association rule and the number of node. The present minimum support is set to be $10 \%$ in order to reduce the number of elements because many nodes with the small influence are generated. 
The hierarchical dendrograms which the first stratum has the association rule regarding the objective functions are constructed for the minimisation of all of the three objective functions. Note that there are severe tradeoffs between the initial gross weight and the other two objective functions, which was already revealed in the optimisation results shown in Figure 3 and those from SOM. And also, since the initial gross weight is the minimisation function while the other two objective functions are maximisation functions, Figure 5 as the result for the minimisation of the initial gross weight is shown as the representative of the generated hierarchical dendrograms. The decoding manner of each node in the present hierarchical dendrogram shown in Figure 5 is explained in the caption of Figure 5.

Figure 5 Hierarchical dendrogram generated by the stratum-type association analysis for the minimisation of the initial gross weight $M_{\mathrm{tot}}(0)$ (see online version for colours)

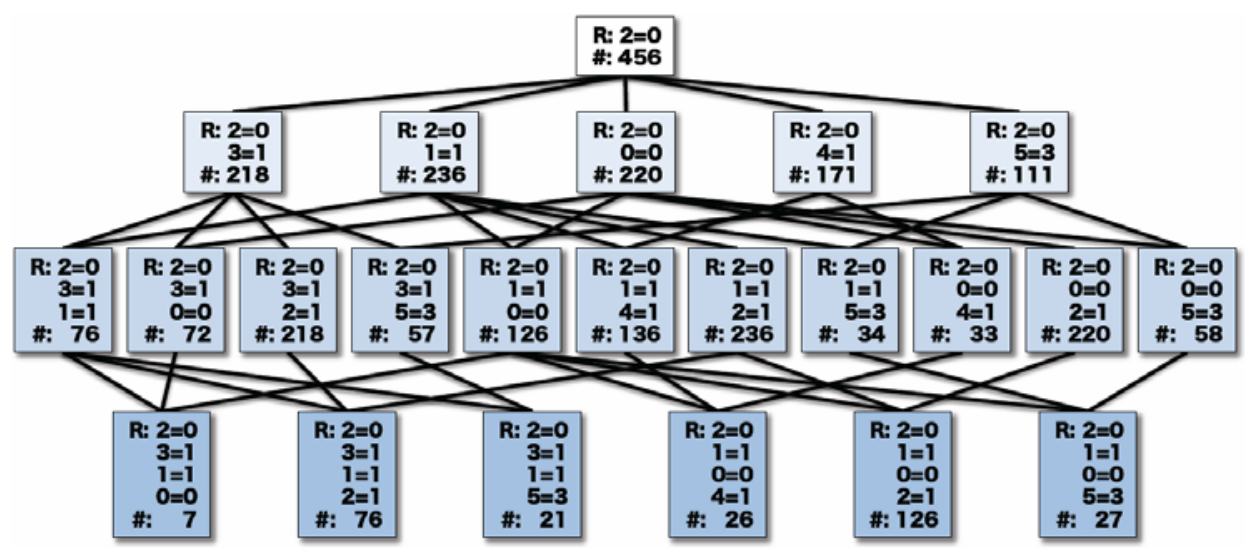

Notes: The present dendrogram is constructed by 23 nodes. The two-line explanations show the rule (which is described as 'the variables' = 'its discretised region') from the top line using 'R:' and the correspondent number of all non-dominated solutions with the rule at the bottom using '\#:'. The variable numbers of 0 to 9 respectively denote obj1, obj2, obj3, dv1, dv2, , , and dv7. Since all variables are discretised into 10 in the present study, the discretised region is described by using the number of 0 to 9 . The top node is in the first stratum and the bottom nodes are in the fourth stratum.

The first stratum has 456 non-dominated solutions (because the total number of 999 is obtained for non-dominated solutions by the optimisation process, the proportion of application is roughly $45.6 \%$ ) which have the attribute with the minimum-value node of $M_{\text {tot }}(0)$. This result indicates that roughly half number of the non-dominated solutions has the small value of $M_{\mathrm{tot}}(0)$.

The second stratum has six nodes with two attributes. Since one attribute is ' $2=0$ ', i.e., the minimum-value node of $M_{\text {tot }}(0)$, the node indicates the tradeoff information when the other attribute is the rule regarding the other objective functions. In contrast, the node indicates the information regarding the effective design variable to minimise $M_{\mathrm{tot}}(0)$ when the other attribute is the rule regarding the design variables. The third node from the left has the rule for $R_{d}$ with the lowest values (the number of non-dominated solutions is 220). This node reveals the severe tradeoff because $R_{d}$ for the $48 \%$ of the non-dominated solutions is in the discretised region for minimum value when $M_{\mathrm{tot}}(0)$ is minimised. The 
second node from the left has the rule for $T_{d}$ with low values (the number of non-dominated solutions is 236). This node also reveals the severe tradeoff because $T_{d}$ for the $52 \%$ of the non-dominated solutions is in the discretised region for minimum value when $M_{\text {tot }}(0)$ is minimised. Furthermore, the first node from the left has the rule for $\dot{m}_{\text {oxi }}(0)$ (dv1) with low values (the number of non-dominated solutions is 218 ). The fourth node from the left is the rule for $L_{\text {fuel }}(\mathrm{dv} 2)$ with low values (the number of non-dominated solutions is 171). These nodes reveal that dv1 and dv2 are essential to restrain $M_{\mathrm{tot}}(0)$. In addition, $\mathrm{dv} 1$ and $\mathrm{dv} 2$ are the significant design variables in order to also reduce the other two objective functions. The first node from the right is the rule for $r_{\text {port }}(0)(\mathrm{dv} 3)$ with low values (the number of non-dominated solutions is 11 as a small number). $r_{\text {port }}(0)(\mathrm{dv} 3)$ indirectly gives an effect because small $r_{\text {port }}(0)(\mathrm{dv} 3)$ gives an effect on restraining the mass flow of oxidiser $\dot{m}_{\text {oxi }}(t)$ although $r_{\text {port }}(0)(\mathrm{dv} 3)$ does not have a direct effect.

The third stratum has 13 nodes with three attributes. Ten nodes of those consist of two rules regarding the objective function and one rule regarding the design variable. Since the knowledge of the second stratum shows the effective design variable for the objective functions, the observation of these ten nodes can be omitted due to the comprehension into the design knowledge from the second stratum. The crucial point in the third stratum is that merely one node exists which has one rule regarding the objective function and two rules regarding the design variables. This result reveals that the correlation between the objective functions is strong and one design variable to depend on each objective function can be narrowed.

The fourth stratum has seven nodes with four attributes. Since the knowledge of the third stratum reveals that the present problem has severe tradeoffs, five nodes have three rules regarding the objective function and one rule regarding the design variable. The fourth node of the left merely has two rules regarding the objective functions and two rules regarding the design variables. However, since the indicated design variables are $\dot{m}_{\text {oxi }}(0)(\mathrm{dv} 1)$ and $r_{\text {port }}(0)(\mathrm{dv} 3)$, the knowledge from the second stratum can be similarly interpreted.

Although the stratum-type association analysis cannot instruct the optimum and pessimum directions, the severeness of tradeoff can be quantitatively shown by using the number of application. In addition, the magnitude of the influence of the design variables on the objective functions can be quantitatively elucidated. Moreover, since the non-dominated solutions to apply to a node are generated, the stratum-type association analysis is also useful for the selection of specific non-dominated solutions in order to perform the data mining for the significant local region in the objective-function space.

\section{Conclusions}

The next-generation single-stage launch vehicle with the hybrid rocket engine of solid fuel and liquid oxidiser in place of the present pure solid-fuel rockets has been conceptually designed by using design informatics in order to contribute to the development of the low cost launch vehicle system and efficient space scientific observation. A SOM generates the bird's-eye visualisation for the correlations among the objective functions and the design variables. Moreover, the stratum-type association analysis is used in order to structurise and visualise the design space. Consequently, the 
design information has been revealed regarding the tradeoffs among the objective functions, the behaviour of the design variables in the design space, and the implication of the design variables for the objective functions from a SOM. Moreover, the hierarchical dendrogram obtained by using the stratum-type association analysis indicates the structure of the design variables in order to improve the objective functions. This methodology is expected to be strongly beneficial for designers.

\section{References}

Arias-Montano, A., Coello, C.A.C. and Mezura-Montes, E. (2012) 'Multiobjective evolutionary algorithms in aeronautical and aerospace engineering', IEEE Transactions on Evolutionary Computation, Vol. 16, No. 5, pp.662-694.

Arthur, W. (2002) 'The emerging conceptual framework of evolutionary developmental biology', Nature, Vol. 415, No. 6873, pp.757-764.

Baker, J.E. (1985) 'Adaptive selection methods for genetic algorithms', Proceedings of the International Conference on Genetic Algorithms and their Applications, pp.101-111, Lawrence Erlbaum Associates.

Chiba, K. (2013) 'Evolutionary hybrid computation in view of design information by data mining', Proceedings on IEEE Congress on Evolutionary Computation, pp.3387-3394, IEEE.

Chiba, K. and Obayashi, S. (2008) 'Knowledge discovery in aerodynamic design space for flyback-booster wing using data mining', Journal of Spacecraft and Rockets, Vol. 45, No. 5, pp.975-987.

Chiba, K., Kanazaki, M., Nakamiya, M., Kitagawa, K. and Shimada, T. (2013) 'Conceptual design of single-stage launch vehicle with hybrid rocket engine for scientific observation using design informatics', Journal of Space Engineering, Vol. 6, No. 1 pp.15-27.

Chiba, K., Kanazaki, M., Nakamiya, M., Kitagawa, K. and Shimada, T. (2014) 'Diversity of design knowledge for launch vehicle in view of fuels on hybrid rocket engine', Journal of Advanced Mechanical Design, Systems, and Manufacturing JAMDSM0023, Vol. 8, No. 3, pp.1-14.

Chiba, K., Makino, Y. and Takatoya, T. (2012) 'Design-informatics approach for intimate configuration of silent supersonic technology demonstrator', Journal of Aircraft, Vol. 49, No. 5, pp.1200-1211.

Deb, K., Pratap, A., Agarwal, S. and Meyarivan, T. (2002) 'A fast and elitist multiobjective genetic algorithm: NSGA-II', IEEE Transactions on Evolutionary Computation, Vol. 6, No. 2, pp.182-197.

Deboeck, G. and Kohonen, T. (1998) Visual Explorations in Finance with Self-Organizing Maps, Springer Finance, London.

Fonseca, C.M. and Fleming, P.J. (1993) 'Genetic algorithms for multiobjective optimization: formulation, discussion and generalization', Proceedings of the Fifth International Conference on Genetic Algorithms, pp.416-423, Morgan Kaufmann.

Gordon, S. and McBride, B.J. (1994) Computer Program for Calculation of Complex Chemical Equilibrium Compositions and Applications I: Analysis, NASA Reference Publication RP-1311, NASA.

Hervas-Martinez, C., Ortiz-Bayer, D. and Garcia-Pedrajas, N. (2002) 'Theoretical analysis of the confidence interval based crossover for real-coded genetic algorithms', The 7th International Conference on Parallel Problem Solving from Nature, LNCS, Vol. 2439, pp.153-161, Springer-Verlag, Berlin Heidelberg.

Hirata, K., Sezaki, C., Yuasa, S., Shiraishi, N. and Sakurai, T. (2011) 'Fuel regression rate behavior for various fuels in swirling-oxidizer-flow-type hybrid rocket engines', AIAA Paper 2011-5677, AIAA. 
Jeong, S., Murayama, M. and Yamamoto, K (2005) 'Efficient optimization design method using kriging model', Journal of Aircraft, Vol. 42, No. 2, pp.413-420.

Karabeyoglu, M.A. (2013) 'Advanced hybrid rockets for future space launch', Proceedings on 5th European Conference for Aeronautics and Space Sciences: EUCASS.

Karabeyoglu, M.A., Altman, D. and Cantwell, B.J. (2002) 'Combustion of liquefying hybrid propellants: part 1, general theory', Journal of Propulsion and Power, Vol. 18, No. 3, pp.610-620.

Keane, A.J. (2006) 'Statistical improvement criteria for use in multiobjective design optimization', AIAA Journal, Vol. 44, No. 4 pp.879-891.

Kohonen, T. (1995) Self-Organizing Maps, Springer, Berlin, Heidelberg.

Kosugi, Y., Oyama, A., Fujii, K. and Kanazaki, M. (2011) 'Multidisciplinary and multi-objective design exploration methodology for conceptual design of a hybrid rocket', AIAA Paper 2011-1634, AIAA.

Oyama, A., Obayashi, S. and Nakamura, T. (2001) 'Real-coded adaptive range genetic algorithm applied to transonic wing optimization', Applied Soft Computing, Vol. 1, No. 3, pp.179-187.

Robic, T. and Filipic, B. (2005) 'DEMO: differential evolution for multiobjective optimization', The 3rd International Conference on Evolutionary Multi-Criterion Optimization, LNCS, Vol. 3410, pp.520-533, Springer-Verlag, Guanajuato, Mexico.

Saraniero, M.A., Caveny, L.H. and Summerfield, M. (1973) 'Restart transients of hybrid rocket engines', Journal of Spacecraft and Rockets, Vol. 10, No. 3, pp.215-217.

Sasaki, D. and Obayashi, S. (2005) 'Efficient search for trade-offs by adaptive range multi-objective genetic algorithms', Journal of Aerospace Computing, Information, and Communication, Vol. 2, No. 1, pp.44-64.

Simurda, L., Zilliac, G. and Zaseck, C. (2013) 'High performance hybrid propulsion system for small satellites', AIAA Paper, pp.2013-3635, AIAA.

Takahashi, M. and Kita, H. (2001) 'A crossover operator using independent component analysis for real-coded genetic algorithms', Proceedings of IEEE Congress on Evolutionary Computation 2001, pp.643-649, IEEE.

Watanabe, S., Chiba, Y. and Kanazaki, M. (2014) 'A proposal on analysis support system based on associate rule analysis for non-dominated solutions', Proceedings on IEEE World Congress on Computational Intelligence, p.E-14855, IEEE, Beijing, China.

Yuasa, S., Shiraishi, N. and Hirata, K. (2012) 'Controlling parameters for fuel regression rate of swirling-oxidizer-flow-type hybrid rocket engine', AIAA Paper 2012-4106, AIAA. 